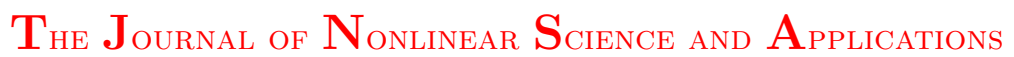
http://www.tjnsa.com

\title{
REMARKS ON REMOTAL SETS IN VETOR VALUED FUNCTION SPACES
}

\author{
M. SABABHEH ${ }^{1}$ AND R. KHALIL ${ }^{2 *}$ \\ Communicated by I. Beg
}

\begin{abstract}
Let $X$ be a Banach space and $E$ be a closed bounded subset of $X$. For $x \in X$ we set $D(x, E)=\sup \{\|x-e\|: e \in E\}$. The set $E$ is called remotal in $X$ if for any $x \in X$, there exists $e \in E$ such that $D(x, E)=\|x-e\|$. It is the object of this paper to give new results on remotal sets in $L^{p}(I, X)$, and to simplify the proofs of some results in 5 .
\end{abstract}

\section{INTRODUCTION AND PRELIMINARIES}

Let $X$ be a Banach space and $E$ be a closed bounded subset of $X$. For $x \in X$ we set $D(x, E)=\sup \{\|x-e\|: e \in E\}$. The set $E$ is called remotal in $X$ if for any $x \in X$, there exists $e \in E$ such that $D(x, E)=\|x-e\|$. The study of remotal sets started almost in the sixties. It turned out that remotal sets have applications in geometry of Banach spaces. However, almost all known results on remotal sets are concerned with the topological properties of such sets. In [5], the problem of what conditions one can impose to ensure that $L^{1}(I, E)$ be remotal in $L^{1}(I, X)$ was studied. The object of this paper is to give new results on remotal sets in $L^{p}(I, X)$, and to present full proofs of certain results in [5]. Further, we introduce the concept of sphere images of sets. We believe that such a concept will be fruitful in the theory of remotal sets. We refer to [1], 2], [3] and [5] for classical results on remotal sets.

Throughout this paper, $I$ denotes the unit interval with the Lebesgue measure, and $L^{p}(I, X)$ is the space of $p$-Bochner integrable functions (equivalence classes) defined on $I$ with values in $X$. The closed unit ball of any Banach space $X$ is denoted by $B[X]$. We let $S(x, r)$ denote the sphere of center $x$ and radius $r$.

Date: Received: January 2009.

* Corresponding author.

2000 Mathematics Subject Classification. 46B20, 41A50, 41A65.

Key words and phrases. Remotal sets, Approximation theory in Banach spaces. 


\section{MAin RESUlts}

2.1. Sphere Reflection of Sets. Let $X$ be a Banach space and $E$ be a closed and bounded set in $X$. For $x \in X \backslash E$, let $D(x, E)=r$, and $S(x, r)=\{y:\|x-y\|=r\}$. We write $S$ for $S(x, r)$. Consider the following map

$$
\varphi_{x}: E \rightarrow X, \varphi_{x}(e)=2 x+2 r \frac{e-x}{\|e-x\|}-e \text {. Then, }
$$

Proposition 2.1. The map $\varphi_{x}$ has the following properties

(i) $\varphi_{x}$ is continuous

(ii) $\varphi_{x}(E)$ is closed and bounded.

(iii) $d(S, E)=\inf \{\|z-e\|: z \in S$ and $e \in E\}=0$.

(iv) $d\left(E, \varphi_{x}(E)\right)=0$

(v) $d\left(x, \varphi_{x}(E)\right)=r$

(vi) $x$ has a farthest point in $E$ if and only if $x$ has a closest element in $\varphi_{x}(E)$.

Proof. The proof of $(i)$ is evident noting that $E$ is closed and bounded. For $(i i)$, suppose that $\varphi_{x}\left(e_{n}\right) \longrightarrow y \in X$ where $e_{n} \in E$. Since $\left\|e_{n}-x\right\|$ is a bounded sequence of real numbers, it must have a convergent subsequence, we may suppose that $\left\|e_{n}-x\right\|$ converges to some real number. Now, it can be shown easily that $e_{n} \rightarrow e \in E$ and hence $y=\varphi_{x}(e)$. Therefore, $\varphi_{x}(E)$ is closed. Since $\left\|x-\varphi_{x}(e)\right\| \leq 3 r$, then $\varphi_{x}(E)$ is bounded.

To prove $(i i i)$, let $e_{n} \in E$ such that $\left\|x-e_{n}\right\| \rightarrow r$. Let $s_{n}=x+r \frac{e_{n}-x}{\left\|e_{n}-x\right\|}$. Clearly $s_{n} \in S$ and $\left\|e_{n}-s_{n}\right\|=\left\|\left(x-e_{n}\right)\left(1-\frac{r}{\left\|x-e_{n}\right\|}\right)\right\| \rightarrow 0$. Hence $d(S, E)=0$.

As for $(i v)$, let $e_{n} \in E$ such that $\left\|x-e_{n}\right\| \rightarrow r$. Then

$$
\left\|e_{n}-\varphi_{x}\left(e_{n}\right)\right\|=\left\|2 x+2 r \frac{e_{n}-x}{\left\|e_{n}-x\right\|}-e_{n}-e_{n}\right\|=2\left\|e_{n}-x\right\|\left|1-\frac{r}{\left\|e_{n}-x\right\|}\right| \rightarrow 0 .
$$

Now, for any $e \in E$,

$$
\begin{aligned}
\left\|x-\varphi_{x}(e)\right\| & =\left\|-x-2 r \frac{e-x}{\|e-x\|}+e\right\| \\
& =2 r-\|e-x\| \geq r,
\end{aligned}
$$

where in the last line, we have used the fact that $D(x, E)=r$. Now, if $\left\|x-e_{n}\right\| \rightarrow r$, we would have $\left\|x-\varphi_{x}\left(e_{n}\right)\right\| \rightarrow r$. This shows that $d\left(x, \varphi_{x}(E)\right)=r$.

Let $D(x, E)=\|x-e\|=r$ for some $e \in E$. Then,

$$
\left\|x-\varphi_{x}(e)\right\|=\left\|2 x-2 r \frac{e-x}{\|e-x\|}-e-x\right\|=\|e-x\|=r .
$$

But from $(v), d\left(x, \varphi_{x}(E)\right)=r$. Hence $x$ has a closest element in $\varphi_{x}(E)$. A similar argument proves the converse. This ends the proof of the proposition.

We should remark that $\varphi_{x}: E \rightarrow \varphi_{x}(E)$ is $1-1$, a fact that one can easily prove.

Definition 2.2. Let $X$ be a Banach space. We say that $X$ has the mirror reflection property if for any closed and bounded set $E \subset X$, and any $x \in X \backslash E$, there exists a closed convex set $G \subset E$, such that $D(x, E)=D(X, G)$ and $\varphi_{x}(G)$ is convex.

Lemma 2.3. Every finite dimensional normed space has the mirror reflection property. 
Proof. Let $X$ be finite dimensional, and $x \in X$. Since $E$ is closed and bounded, then it is compact. But any compact set is remotal. Thus if $D(x, E)=r$, then there exists $e \in E$ such that $\|x-e\|=D(x, E)=r$. But then $\varphi_{x}(e)=e$. Since $\{e\}$ is convex, the set $G=\{e\}$ satisfies $\varphi_{x}(G)$ is convex.

Now, the closed unit ball of a subspace $Y$ of the Banach space $X$ is not necessarily remotal. However,

Theorem 2.4. Let $Y$ be a reflexive subspace of the Banach space $X$ that has the mirror reflection property. Then $B[Y]$ is remotal in $X$.

Proof. Let $x \in X$. If $x \in B[Y]$ then, trivially, $x$ has a farthest element in $B[Y]$, see Lemma 2.12 below. So we may assume that $x \notin B[Y]$ and that $F(x, B[Y])=r>$ 0 . Let $E \subset B[Y]$ be such that $E$ is convex, closed and $\hat{E}=\varphi_{x}(E)$ is convex with $D(x, B[Y])=D(x, E)$. Such an $E$ exists because $X$ has the mirror reflection property. Now $d\left(x, \varphi_{x}(E)\right)=D(x, E)$ and $d(E, S)=d\left(S, \varphi_{x}(E)\right)=d\left(E, \varphi_{x}(E)\right)=0$. Further $\operatorname{span}\left(\varphi_{x}(E)\right)$ is reflexive because $E$ is a subset of a reflexive spaces $Y$ and $\operatorname{span}\left(\varphi_{x}(E)\right)=$ $\operatorname{span}(E \cup\{x\})$. Let $\left(z_{n}\right) \subset \varphi_{x}(E)$ be such that $\left\|x-z_{n}\right\| \longrightarrow r, z_{n}=\varphi_{x}\left(e_{n}\right), e_{n} \in E$. But

$$
\begin{aligned}
\left\|x-\varphi_{x}\left(e_{n}\right)\right\| & =\left\|x+2 r \frac{e_{n}-x}{\left\|e_{n}-x\right\|}-e_{n}\right\| \\
& =\left\|x\left(1-2 r /\left\|e_{n}-x\right\|\right)-e_{n}\left(1-2 r /\left\|e_{n}-x\right\|\right)\right\| \\
& =2 r-\left\|x-e_{n}\right\| .
\end{aligned}
$$

Since $\left\|x-\varphi_{x}\left(e_{n}\right)\right\| \longrightarrow r$ we see that $\left\|x-e_{n}\right\| \longrightarrow r$. Consequently $\left\|\varphi_{x}\left(e_{n}\right)-e_{n}\right\| \longrightarrow 0$. This follows from

$$
\begin{aligned}
\varphi_{x}\left(e_{n}\right)-e_{n} & =2 x+2 r \frac{e_{n}-x}{\left\|e_{n}-x\right\|}-2 e_{n} \\
& =2 r \frac{e_{n}-x}{\left\|e_{n}-x\right\|}-2\left(e_{n}-x\right) \\
& =\left(e_{n}-x\right)\left[\frac{2 r}{\left\|e_{n}-x\right\|}-2\right] .
\end{aligned}
$$

Hence, $\left\|\varphi_{x}\left(e_{n}\right)-e_{n}\right\|=\left\|e_{n}-x\right\|\left[\frac{2 r}{\left\|e_{n}-x\right\|}-2\right] \longrightarrow 0$. Now $\varphi_{x}\left(e_{n}\right)$ is a bounded sequence in a closed convex set $\varphi_{x}(E)$ in a reflexive space $\tilde{Y}$. Hence, with no loss of generality, we can assume the existence of $z$ such that $\varphi_{x}\left(e_{n}\right) \longrightarrow z$ weakly, Alaoglu theorem guarantees the compactness of $\varphi_{x}(E)$ in the $w^{*}$-topology. Also, we may assume that $e_{n} \longrightarrow p$ in the $w^{*}$-topology. So

$$
\begin{aligned}
\left|\left\langle z-p, y^{*}\right\rangle\right| & =\lim _{n \rightarrow \infty}\left|\left\langle\varphi_{x}\left(e_{n}\right)-e_{n}, y^{*}\right\rangle\right| \\
& \leq \lim _{n \rightarrow \infty}\left\|\varphi_{x}\left(e_{n}\right)-e_{n}\right\|\left\|y^{*}\right\|=0 .
\end{aligned}
$$

Since this is true for all $y^{*} \in \tilde{Y}$, we must have $p=z$. However, since both $E$ and $\varphi_{x}(E)$ are closed and convex, then $z \in \varphi_{x}(E)$ and $p \in E$. Therefore,

$$
z=p \in \varphi_{x}(E) \cap E \subset S .
$$

But this means that $p$ is a farthest element in $E$ from $x$ and we are done. 
2.2. Remotal sets in $L^{p}(I, X)$. Let $I$ be the unit interval with Lebesgue measure. $L^{p}(I, X)$ denotes the space of $p$-Bochner integrable functions (equivalence classes) on $I$ with values in $X$. One of the problems in the theory of existence of farthest points is "If $E$ is remotal in $X$, must $\mathrm{E}^{p}(I, E)=\left\{f \in L^{p}(I, X): f(t) \in E\right.$ a.e $\}$ be remotal in $L^{p}(I, X)$ ?". Not much results are known in that direction. In [5], some results were given on that problem. In this section we will give simpler proofs of some of the results in [5], and present new results on remotality of $L^{p}(I, E)$.

First, we prove the following distance formula:

Theorem 2.5. Let $E$ be any closed bounded set in a Banach space $X$. Then, for $f \in L^{p}(I, X)$,

$$
\sup _{g \in L^{p}(I, E)}\|f-g\|_{p}=\left(\int_{I} \sup _{e \in E}\|f(t)-e\|^{p} d t\right)^{1 / p}, \text { for } 1 \leq p<\infty .
$$

Proof. For $g \in L^{p}(I, G)$ we have

$$
\|f-g\|_{p}^{p}=\int_{I}\|f(t)-g(t)\|^{p} d t \leq \int_{I} \sup _{e \in E}\|f(t)-e\|^{p} d t,
$$

hence, on taking supremum over $g \in L^{p}(I, E)$ we get

$$
\sup _{g \in L^{p}(I, E)}\|f-g\|_{p} \leq\left(\int_{I} \sup _{e \in E}\|f(t)-e\|^{p} d t\right)^{1 / p} .
$$

For the reverse inequality, let $\epsilon>0$ be given and let $\varphi=\sum_{i=1}^{n} y_{i} \chi_{A_{i}}$ be a simple function in $L^{p}(I, X)$ such that $\|\varphi-f\|_{p}<\epsilon$. Here, $y_{i} \in X$ and the $A_{i}$ are subsets of $I$ that may be assumed to be disjoint and non-empty. For each $y_{i}$ let $e_{i} \in E$ be such that

$$
\left\|y_{i}-e_{i}\right\|^{p}>\sup _{e \in E}\left\|y_{i}-e\right\|^{p}-\frac{\epsilon}{n \mu\left(A_{i}\right)}
$$

Now, let $w=\sum_{i=1}^{n} e_{i} \chi_{A_{i}}$. Then $w \in L^{p}(I, E)$ and

$$
\|\varphi-w\|_{p} \leq\|\varphi-f\|_{p}+\|f-w\|_{p} \leq\|f-w\|_{p}+\epsilon .
$$

Consequently,

$$
\begin{aligned}
\left(\|f-w\|_{p}+\epsilon\right)^{p} & \geq\|\varphi-w\|_{p}^{p}=\int_{I}\|\varphi(t)-w(t)\|^{p} d t \\
& =\sum_{i=1}^{n} \int_{A_{i}}\|\varphi(t)-w(t)\|^{p} d t \\
& =\sum_{i=1}^{n}\left\|y_{i}-e_{i}\right\|^{p} \mu\left(A_{i}\right) \\
& >\sum_{i=1}^{n}\left[\sup _{e \in E}\left\|y_{i}-e\right\|^{p}-\frac{\epsilon}{n \mu\left(A_{i}\right)}\right] \mu\left(A_{i}\right) \\
& =\sum_{i=1}^{n} \sup _{e \in E}\left\|y_{i}-e\right\|^{p} \mu\left(A_{i}\right)-\epsilon .
\end{aligned}
$$


Therefore,

$$
\begin{aligned}
\left(\|f-w\|_{p}+\epsilon\right)^{p}+\epsilon & >\sum_{i=1}^{n} \int_{A_{i}} \sup _{e \in E}\left\|y_{i}-e\right\|^{p} d t \\
& =\int_{I} \sum_{i=1}^{n} \sup _{e \in E}\left\|y_{i}-e\right\|^{p} \chi_{A_{i}}(t) d t \\
& =\int_{I} \sup _{e \in E}\|\varphi(t)-e\|^{p} d t \\
& \geq \int_{I} \sup _{e \in E}|\|f(t)-e\|-\|\varphi(t)-f(t)\||^{p} d t \\
& \geq \int_{I}\left|\sup _{e \in E}\|f(t)-e\|-\|\varphi(t)-f(t)\|\right|^{p} d t .
\end{aligned}
$$

Let $h(t)=\sup _{e \in E}\|f(t)-e\|$ and $k(t)=\|\varphi(t)-f(t)\|$. These are real valued functions. Let us for convenience denote by $\|h\|_{p(\mathbb{R})}$ the $L^{p}$ norm of the real valued function $h$. Keeping this in mind, the last integral becomes $\|h-k\|_{p(\mathbb{R})}^{p}$. Whence,

$$
\begin{aligned}
{\left[\left(\|f-w\|_{p}+\epsilon\right)^{p}+\epsilon\right]^{1 / p} } & \geq\|h-k\|_{p(\mathbb{R})} \\
& \geq\|h\|_{p(\mathbb{R})}-\|k\|_{p(\mathbb{R})} \\
& \geq\left(\int_{I} \sup _{e \in E}\|f(t)-e\|^{p}\right)^{1 / p}-\epsilon .
\end{aligned}
$$

Thus,

$$
\begin{aligned}
\left(\int_{I} \sup _{e \in E}\|f(t)-e\|^{p}\right)^{1 / p} & \leq\left[\left(\|f-w\|_{p}+\epsilon\right)^{p}+\epsilon\right]^{1 / p}+\epsilon \\
& \leq\left[\left(\sup _{g \in L^{p}(I, G)}\|f-g\|_{p}+\epsilon\right)^{p}+\epsilon\right]^{1 / p}+\epsilon,
\end{aligned}
$$

where we have used the fact that $w \in L^{p}(I, G)$. On letting $\epsilon \longrightarrow 0$ we get

$$
\left(\int_{I} \sup _{e \in E}\|f(t)-e\|^{p}\right)^{1 / p} \leq \sup _{g \in L^{p}(I, G)}\|f-g\|_{p},
$$

as required.

Corollary 2.6. Let $X$ be a Banach space and $E$ be a closed bounded subset of $X$. Then, for $1 \leq p<\infty, g \in L^{p}(I, E)$ is farthest from $f \in L^{p}(I, X)$ if and only if, for almost all $t \in I, g(t)$ is farthest in $E$ from $f(t)$.

Proof. Suppose that $g$ is farthest from $f$ in $L^{p}(I, E)$. By the above theorem we have

$$
\int_{I}\|f(t)-g(t)\|^{p} d t=\int_{I} \sup _{e \in E}\|f(t)-e\|^{p} d t
$$

which means

$$
\int_{I}\left(\sup _{e \in E}\|f(t)-e\|^{p}-\|f(t)-g(t)\|^{p}\right) d t=0 .
$$

But the integrand is non-negative because $g \in L^{p}(I, E)$, hence $\|f(t)-g(t)\|=\sup _{e \in E} \| f(t)-$ $e \|$ for almost all $t \in I$. This means that for almost all $t \in I, g(t)$ is farthest in $E$ from $f(t)$. The other part of the theorem is trivial. 
As a corollary of this corollary we get:

Corollary 2.7. Suppose that $L^{p}(I, E)$ is remotal in $L^{p}(I, X)$ for some $1 \leq p<\infty$, where $E$ is a closed bounded subset of the Banach space $X$, then $E$ itself is remotal in $X$.

Proof. Let $x \in X$ and define $f(t)=x$. Then $f \in L^{p}(I, X)$. Therefore, there exists $g \in L^{p}(I, E)$ such that $g$ is farthest from $f$. By the above corollary, $g(t)$ is farthest from $f(t)$ for almost all $t$. But $f(t)=x$ for all $t$. Hence, there exists a $t \in E$ such that $g(t)$ is farthest in $E$ from $x \in X$.

For a remotal set $E \subset X$, the map $H$ that maps any element $x$ to $F(x, E):=\{e \in$ $E:\|x-e\|=D(x, E)\}$ is a multi-valued map in general. Hence for $f \in L^{p}(I, X)$, the map $H \circ f$ is a multi-valued map from $I$ into $E$.

Before proceeding, we remind the reader of some facts regarding multi-valued mappings and related concepts: Let $S$ be a measurable space and $X$ a Banach space. A function $f: S \longrightarrow X$ is said to be strongly measurable if there exists a sequence $\left\{f_{n}\right\}$ of simple functions such that

$$
\lim _{n \rightarrow \infty}\left\|f_{n}(s)-f(s)\right\|=0
$$

almost everywhere. On the other hand, we say that $f$ is measurable in the classical sense if $f^{-1}(K)$ is measurable, in $S$, for every closed set $K$ in $X$. It is always true that strong measurability implies measurability in the classical sense, provided that $S$ is a complete measure space, but not vice versa, see [4] p.114. However, if $f: S \longrightarrow X$ is measurable in the classical sense and has essentially separable range, then $f$ is strongly measurable, see [4], p.114. A multi-valued mapping $F: I \longrightarrow X$ is said to be measurable if $F^{-1}(K)$ is measurable for every closed subset $K$ of $X$. Here, $F^{-1}(K)=\{t \in I: F(t) \cap K \neq \phi\}$. A measurable (in the classical sense) function $f$ may be extracted from a measurable multi-valued mapping $F: I \longrightarrow X$, where $X$ is a separable Banach space, provided that $F(t)$ is a closed subset of $X$ for each $t \in I$, and such that $f(t) \in F(t)$ for each $t \in I$, consult [6], p.289.

Lemma 2.8. If $E$ is a finite set in $X$, then the map $H \circ f$ has a measurable selection, where $f: I \longrightarrow X$ is measurable.

Proof. Since $E$ is finite, then every subset of is $E$ finite and hence closed. So $H^{-1}(G)$ is closed for any closed subset $G$ of $E$. Indeed, suppose that $x_{n} \longrightarrow x$ where $x_{n} \in H^{-1}(G)$ and $x \in X$. Since $x_{n} \in H^{-1}(G), H\left(x_{n}\right) \cap G \neq \phi$. Let $y_{n} \in H\left(x_{n}\right) \cap G$, then we may assume that $y_{n} \longrightarrow y \in G$ because $G$ is finite. We assert that $y \in H(x)$ :

$$
\begin{aligned}
\|x-y\| & =\lim _{n \rightarrow \infty}\left\|x_{n}-y_{n}\right\| \\
& \geq \lim _{n \rightarrow \infty}\left\|x_{n}-w\right\| \forall w \in E \\
& =\|x-w\| \forall w \in E .
\end{aligned}
$$

Consequently, $y \in H(x)$ and $x \in H^{-1}(\{y\}) \subset H^{-1}(G)$. This shows that $H^{-1}(G)$ is closed.

Since $f$ is measurable, then $\left.f^{-1}\left(H^{-1}(G)\right)\right)$ is measurable. Hence, $H \circ f$ is a measurable multi-valued function. Hence, [6] page $289, H \circ f$ has a measurable selection. 
Theorem 2.9. Let $E$ be a finite set in the Banach space $X$. Then $L^{1}(I, E)$ is remotal in $L^{1}(I, X)$.

Proof. Let $f \in L^{1}(I, X)$. Then $H \circ f(t)$ is the set of the farthest point from $f(t)$ in $E$. By Lemma 2.8, $H \circ f$ has a measurable selection, $g$, say. Since $g \in L^{1}(I, E)$, then $L^{1}(I, E)$ is remotal in $L^{1}(I, X)$, and $g \in F\left(f, L^{1}(I, E)\right)$.

Theorem 2.10. Let $E$ be a closed bounded set in $X$ such that the span of $E$ is a finite dimensional subspace of $X$. Then $L^{1}(I, E)$ is remotal in $L^{1}(I, X)$.

Proof. We will show that the map $H \circ f$ has a measurable selection for any $f \in$ $L^{1}(I, X)$. First we show that $H$ is a closed valued map. Indeed, if $H(x)$ is a finite set, then it is closed. If $H(x)$ is not finite, then let $y$ be a limit point of $H(x)$. Then there is a sequence $y_{n} \in H(x)$ such that $y_{n} \rightarrow y$. Then $\left\|x-y_{n}\right\| \geq\|x-e\|$ for all $e \in E$. But then taking the limit, we get $\|x-y\| \geq\|x-e\|$ for all $e \in E$. Hence $H(x)$ is closed. Hence $H$ is a closed valued map, and consequently, $H \circ f$ is a closed multi-valued map.

Now, let $B$ be a closed set in $E$, and $A=H^{-1}(B)$. Let $x_{n} \in A$, and $x_{n} \rightarrow x$. We claim that $x \in A$. This is equivalent to $H(x) \cap B \neq \phi$. Choose a sequence $y_{n} \in H\left(x_{n}\right) \cap B$. Since $B$ is compact, then $y_{n}$ has a subsequence that converges to some $y$ in $B$. With no loss of generality we can assume that $y_{n} \rightarrow y$. Now, $\left\|x_{n}-y_{n}\right\| \geq\left\|x_{n}-e\right\|$ for all $e \in E$. Taking the limit of both sides to get $\|x-y\| \geq\|x-e\|$ for all $e \in E$. Hence $H(x) \cap B \neq \phi$. So $A$ is a closed set. But it follow then that $H \circ f$ is measurable closed valued map. Hence it has a measurable selection, $g$, say.

Now, $\|f(t)-g(t)\| \geq\|f(t)-h(t)\|$ for all $h \in L^{1}(I, E)$. Hence $\|f-g\|_{1} \geq\|f-h\|_{1}$ for all $h \in L^{1}(I, E)$, and $L^{1}(I, E)$ is remotal in $L^{1}(I, X)$. This ends the proof.

In the following two theorems, we give a relation between remotality in $L^{1}(I, X)$ and $L^{p}(I, X)$ for $1<p<\infty$.

Theorem 2.11. Let $E$ be a closed bounded subset of the Banach space $X . L^{1}(I, E)$ is remotal in $L^{1}(I, X)$ if, and only if, $L^{p}(I, E)$ is remotal in $L^{p}(I, X), 1<p<\infty$.

Proof. Suppose that $L^{1}(I, E)$ is remotal in $L^{1}(I, X)$ and let $f \in L^{p}(I, X)$. Since $I$ is of finite measure, $f \in L^{1}(I, X)$. Let $g \in L^{1}(I, E)$ be such that

$$
\|f-g\|_{1} \geq\|f-h\|_{1} \forall h \in L^{1}(I, E) .
$$

By Corollary 2.6,

$$
\|f(t)-g(t)\| \geq\|f(t)-y\|
$$

for almost every $t \in I$ and for all $y \in E$. In particular, if $w \in L^{p}(I, E)$ then, for almost every $t$,

$$
\|f(t)-g(t)\| \geq\|f(t)-w(t)\|
$$

Whence,

$$
\|f-g\|_{p} \geq\|f-w\|_{p} \forall w \in L^{p}(I, E) .
$$

This shows that $L^{p}(I, E)$ is remotal in $L^{p}(I, X)$.

Now assume that $L^{p}(I, E)$ is remotal in $L^{p}(I, X)$ for some $1<p<\infty$. Let $f \in L^{1}(I, X)$ and define, for $n \in \mathbb{N}$,

$$
E_{n}=\{t \in I:\|f(t)\| \leq n\}
$$


Set $f_{n}=f \chi_{E_{n}}$ where $\chi_{E_{n}}$ is the characteristic function of $E_{n}$. Since $f_{n}$ is bounded and $f \in L^{1}(I, X)$ we conclude that $f_{n} \in L^{p}(I, X)$. Hence, functions $g_{n} \in L^{p}(I, E)$ exist such that, for each $n \in \mathbb{N}$,

$$
\left\|f_{n}(t)-g_{n}(t)\right\| \geq\left\|f_{n}(t)-h(t)\right\|, \forall h \in L^{1}(I, E) .
$$

Consequently,

$$
\left\|f_{n}-g_{n}\right\|_{1} \geq\left\|f_{n}-h\right\|_{1}, \forall h \in L^{1}(I, E)
$$

It is clear that $f_{n} \longrightarrow f$ point-wise since $f_{n+1}=f_{n}$ on $E_{n}$ and that $\cup_{n} E_{n}=I$. Now

$$
\begin{aligned}
\left\|f_{n+1}-g_{n+1}\right\|_{1} & =\int_{I}\left\|f_{n+1}(t)-g_{n+1}(t)\right\| d t \\
& =\int_{E_{n}}\left\|f_{n+1}(t)-g_{n+1}(t)\right\| d t+\int_{I \backslash E_{n}}\left\|f_{n+1}(t)-g_{n+1}(t)\right\| d t .
\end{aligned}
$$

But on $E_{n}, f_{n}=f_{n+1}$. Therefore,

$$
\left\|f_{n+1}-g_{n+1}\right\|_{1}=\int_{E_{n}}\left\|f_{n}(t)-g_{n+1}(t)\right\| d t+\int_{I \backslash E_{n}}\left\|f_{n+1}(t)-g_{n+1}(t)\right\| d t .
$$

But, for almost all $t \in E_{n},\left\|f_{n}(t)-g_{n}(t)\right\| \geq\left\|f_{n}(t)-g_{n+1}(t)\right\|$. Therefore, $\| f_{n}(t)-$ $g_{n}(t)\|=\| f_{n}(t)-g_{n+1}(t) \|$ for almost all $t \in E_{n}$. Thus the $g_{n+1}$ can be chosen so that $g_{n+1}=g_{n}$ on $E_{n}$. Hence $g_{n}$ converges point-wise to some function, say $g \in L^{1}(I, E)$. From (2.1) we see that, for almost all $t \in I,\left\|f_{n}(t)-g_{n}(t)\right\| \geq\left\|f_{n}(t)-h(t)\right\|, \forall h \in$ $L^{1}(I, E)$. On taking the limit as $n \longrightarrow \infty$ we get, for almost all $t \in I$,

$$
\|f(t)-g(t)\| \geq\|f(t)-h(t)\|, \forall h \in L^{1}(I, E)
$$

and consequently $g$ is a farthest element in $\left.L^{(} I, E\right)$ form $f \in L^{1}(I, X)$. This shows that $L^{1}(I, E)$ is remotal in $L^{1}(I, X)$ and the proof is complete.

We conclude this section with the following question:

Problem: Let $E$ be a closed bounded set in a Banach space $X$ such that $\overline{\operatorname{span}(E)}$ is reflexive. Must $L^{1}(I, E)$ be remotal in $L^{1}(I, X)$ ?

\subsection{Further Results.}

Lemma 2.12. Let $Y$ be any Banach space and let $B[Y]$ be its unit ball, then $B[Y]$ is remotal in $Y$.

Proof. Let $y \in Y$. Put $\hat{y}=-\frac{y}{\|y\|}$ if $y \neq 0$ and $\hat{0}=b$ where $b$ is any element with $\|b\|=1$. It is clear that $b$ is a farthest point in $B[Y]$ from 0 . Now, for $y \neq 0$,

$$
\mid y-\hat{y}\|=\| y+\frac{y}{\|y\|}\|=1+\| y \|
$$

but for $x \in B[Y]$ we have

$$
\|y-x\| \leq\|y\|+\|x\| \leq\|y\|+1=\|y-\hat{y}\| .
$$

That is, $\hat{y}$ is a farthest point in $B[Y]$ from $y$.

Corollary 2.13. Let $Y$ be any Banach space and Let $B^{\prime}[Y]$ be any ball in $Y$. Then $B^{\prime}[Y]$ is remotal in $Y$. 
Proof. The result follows from the more general statement which says: if $E$ is remotal in $Y$ then $E+y^{\prime}$ and $r E$ are remotal in $Y$ for any $y^{\prime} \in Y$ and any $r \in \mathbb{R}$. For if $e \in E$ is farthest in $E$ from $y-y^{\prime} \in Y$ then $e+y^{\prime}$ is a farthest element in $E+y^{\prime}$ from $y$. Also, if $e \in E$ is farthest from $\frac{1}{r} y$ then $r e \in E+y^{\prime}$ is farthest from $y$. Observe that the case $r=0$ is the trivial case.

Lemma 2.14. Let $Y$ be a 1-summand Banach space of another Banach space $X$. Then $B[Y]$, the unit ball of $Y$, is remotal in $X$.

Proof. Suppose that $X=Y \oplus_{1} W$ so that $x=y+w$ implies $\|x\|=\|y\|+\|w\|$, here $x \in X, y \in Y$ and $w \in W$. If $x \in X$ let $y \in Y$ and $w \in W$ be such that $x=y+w$ and let $\hat{y}$ be a farthest element in $B[Y]$ from $y$. Then

$$
\begin{aligned}
\|x-\hat{y}\| & =\|y-\hat{y}+w\| \\
& =\|y-\hat{y}\|+\|w\| \\
& \geq\|y-z\|+\|w\| \text { for all } z \in B[Y] \\
& =\|x-z\| .
\end{aligned}
$$

Consequently, $\hat{y}$ is a farthest element in $B[Y]$ from $x=y+w$.

Lemma 2.15. Let $Y$ be any Banach space, then $L^{1}(I, B[Y])$ is remotal in $L^{1}(I, Y)$.

Proof. Let $f \in L^{1}(I, Y)$. Define

$$
\hat{f}(t)=\left\{\begin{array}{cc}
\frac{-f(t)}{\|f(t)\|}, & f(t) \neq 0 \\
b, & f(t)=0
\end{array},\right.
$$

where $b$ is any fixed element with $\|b\|=1$. Then $\hat{f} \in L^{1}(I, B[Y])$. Moreover, $\hat{f}(t)$ is a farthest element in $B[Y]$ from $f(t)$, this follows from lemma 2.12 , That is

$$
\|f(t)-\hat{f}(t)\| \geq\|f(t)-g(t)\| \text { for all } g \in L^{1}(I, B[Y])
$$

which means

$$
\|f-\hat{f}\|_{1} \geq\|f-g\|_{1} \text { for all } g \in L^{1}(I, B[Y]) .
$$

This completes the proof of the lemma.

Now we are ready to prove the following result which describes some remotal sets in functions spaces.

Theorem 2.16. Let $Y$ be a 1-summand Banach space in a Banach space $X$. Then $L^{1}(I, B[Y])$ is remotal in $L^{1}(I, X)$.

Proof. Let $f \in L^{1}(I, X)$, then $f(t)=g(t)+h(t)$ where $g(t) \in Y$ and $h(t) \in W$. Let $P: X \longrightarrow Y$ be the projection defined by $P(y+w)=y$. Now, $g(t)=P(f(t))$ and hence, $g$ is measurable because $P$ is continuous. In a similar way, $h$ is measurable. Therefore, $\|f\|_{1}=\|g\|_{1}+\|h\|_{1}$, that is, $g \in L^{1}(I, Y)$ and $h \in L^{1}(I, W)$. Let $\hat{g}$ be a farthest element in $L^{1}(I, B[Y])$ from $g$. Now,

$$
\begin{aligned}
\|f-\hat{g}\|_{1} & =\|g-\hat{g}\|_{1}+\|h\|_{1} \\
& \geq\|g-r\|_{1}+\|h\|_{1} \text { for all } r \in L^{1}(I, B[Y]) \\
& =\|f-r\| \text { for all } r \in L^{1}(I, B[Y])
\end{aligned}
$$

which ends the proof. 


\section{REFERENCES}

1. Asplund, E., Farthest points in reflexive locally uniformly rotund Banach spaces, Israel J. Math. 4(1966), pp.213-216. 1

2. Baronti, M. and Papini, P., Remotal sets revisited, Taiwanese J. Math. 5(2001), pp.357-373. 1

3. Boszany, A., A remark on uniquely remotal sets in $C(K, X)$, Period.Math.Hungar. 12(1981), pp.11-14. 1

4. Cheney, E. and W. Light, Lecture notes in Mathematics, Springer-Verlag Berlin Heidelberg, 1985. 2.2

5. Khalil, R. and Al-Sharif, Sh., Remotal sets in vector valued function spaces, Scientiae Mathematicae Japonica, 63, No. 3(2006), pp.433-441. (document), 1. 2.2

6. Rolewicz, S., Functional analysis and control theory, D.Reidel publishing company, 1986. 2.2. 2.2

1 Department of Science and Humanities, Princess Sumaya University For Technology, Al Jubaina, Amman 11941, Jordan.

E-mail address: sababheh@yahoo.com

2 Department of Mathematics, Jordan University, Al Jubaiha, Amman 11942, JORDAN.

E-mail address: roshdi@ju.edu.jo 